\title{
SMP2-based Architecture for Navigation Satellite System Simulation
}

\author{
Hou Hongtao*, Li Qun and Wang Chao \\ College of Information Systems and Management, National University of Defense Technology, 410073 ChangSha, HuNan, China
}

Received: 3 Mar. 2013, Revised: 6 Jul. 2013, Accepted: 7 Jul. 2013

Published online: 1 Nov. 2013

\begin{abstract}
The Performance Simulation for the Global Navigation Satellite System (GNSS), which can be used in the performance simulation and evaluation analysis of the Service Volume Segment of the GNSS is a part of the critical algorithm test and system index analysis for the GNSS. In this article, the requirements and features of the GNSS are analyzed firstly, and the method of performance simulation for the GNSS is studied. Then, the system operation architecture and model integration architecture based on compassable simulation based on Simulation Model Portability (SMP2) is proposed. The system architecture consists of model design, development, integrating, executing and analysis, and can be used in simulation for different types of analyses such as those on visibility, coverage, geometry, Dilution of Precision (DOP), Navigation System Performance (NSP), and the availability and continuity of the Navigation System. Finally, the regional performance of the GALILEO Navigation Satellite System in China is analyzed based on the architecture and method, which can fit the features and satisfy the requirements of GNSS simulation.
\end{abstract}

Keywords: Navigation Satellite System Simulation, Simulation Model Portability Standard, Model Integration Framework, Simulation Model Reuse

\section{Introduction}

The Global Navigation Satellite System (GNSS) has an increasingly critical function in human life by facilitating network communications [9]. It can provide all-weather positioning, velocity measurement, and timing for the surface of the Earth and near-earth space users, and can be used widely for both military and civil purposes. The GNSS consists of a satellite subsystem, rocket system, operation control system, users subsystem, launching site subsystem, and TT\&C subsystem. It is a space engineering system with a large scale and complex structure. To support the system integration, functional verification, performance validation and analysis of the GNSS, it is necessary to build a model and simulate the behavior and processes of the system using modern computer simulation techniques and methods.

At present, four GNSS have been built or are being built around the world: GPS, GLONASS, COMPASS, and GALILEO. Simulation is crucial to the process of constructing the GNSS. Given that the simulation for the GNSS depends on integrating a multi-domain model and building a complete circuit of the navigation satellite system, scaling and integration is more difficult. As of the late 1990s, the US military began its large-scale simulation by using the distributed interactive simulation technology for GPS. In 2003, the Simulation Model Portability Standard (SMP) was developed by the European Space Agency (ESA) [4,7], which encountered difficulties in multi-domain model integration and analysis during the design of GALILEO, and supported the integrating models for GALILEO and a series of aeronautical engineering projects. Simulation was also used in the engineering construction of China's COMPASS [9].

The performance simulation for the GNSS was mainly implemented in the performance simulation and evaluation analysis of the Service Volume Segment of the Navigation Satellite System [8]. Given the multi-organizational development of the complex features of the navigation system in various fields, the key question in reducing the difficulty of system development and decreasing the research cycle for multi-domain modeling is which method to reuse and integrate in the heterogeneous simulation models in different fields.

\footnotetext{
*Corresponding author e-mail: houhongtao@ nudt.edu.cn
} 


\section{Simulation Model Portability Standard}

Good portability of the navigation simulation model is critical to rapid and effective integration for the performance simulation of the GNSS $[2,15,16]$. The High Level Architecture (HLA) method is employed to solve the interoperability problem among heterogeneous simulation systems. If the performance simulation of the GNSS in coupling between models is extremely complex, the HLA to integrate the models. However, the performance and efficiency of the simulation will be significantly reduced, and the problem of types, computational complexity of GNSS analysis will not be solved, and the mass simulation will not be realistic. The description of simulation model will be normalized and standardized, which is a basic requirement for the development of model integration and reuse. The Simulation Model Portability Standard (SMP) [17] is developed to improve the portability, maintainability, and reusability of modeling and simulation specification by the ESA since 2003. The SMP specification involved numerous advanced approaches in the field of software engineering and simulation; extracted metadata information from the simulation model; represented the model with the Platform Independent Model (PIM) based on the meta model; and developed simulator components, service components, model components, and interfaces between them. The SMP2 is the current version, and the ECSS E-40-07 specification will be the next version of the SMP $[3,14]$.

The separation of the simulation engine and the model is one of the basic methods for simplifying the modeling work, easy reuse, and integration model during modeling [5,6,10-12]. Based on this, the SMP2 has introduced three design ideas:

1.Separation simulator component and simulation service component. The simulator component is used to integrate the model components and simulation service components, and the simulation service component provides basic simulation service to support the model components and simulation service components, including log service, time management service, and scheduling service. B1y defining the standard interface of SMP2 components, SMP2 can promote the portability of the model in different simulation engines.

2.Separation Platform Independent Model (PIM) and Platform Specific Model (PSM). The PIM is used to describe the type, instance assembly, and scheduling of the models, and does not depend on the platform. The PSM is used to describe the simulation models depending on the special simulation language, and the PIM can be transformed into the PSM by using mapping specification. When a new platform technology appears, we can easily achieve the portability of the models by developing the transformation rules of mapping from the PIM to the PSM.

3.Separation Design-Time Component (DTC) and Run-Time Component (RTC). The DTC is used to describe the model's structure, such as the interface and behavior function. The RTC is used to describe the assembly information of models in some simulation applications, such as field values and initialization values. This approach can support the assembly of models in the different simulation applications.

There are four forms of SMP2 simulation model components: 1) the PIM-DTC component is described by Catalogue of Simulation Model Definition Language (SMDL), and will be saved as the model's design file by the XML format; 2) the PIM-RTC component is described by the Assembly of SMDL, and will be saved as the model's assembly file by the XML format; 3) the PSM-DTC component is described by a high-level programming language, such as $\mathrm{C}++$, and will be generated as a binary model library (dynamic link library); and 4) the PSM-RTC component will instantiate the binary model library, then establish the connections between the attribute values and instance of the models by the model's assembly file. The SMP2 standard consists of the SMP2 component model, meta model, $\mathrm{C}++$ mapping mechanism, and the user's manual $[1,4,7]$.

As a typical simulation of the multi-organization development and multi-domain integration, the performance simulation of the GNSS inevitably requires the model to have good reusability and portability. The model development and integration method based on the SMP2 specification can be used in the research of the performance simulation system of the GNSS.

\section{Models and Analysis of GNSS}

The performance simulation of the GNSS simulates and evaluates the performance of the GNSS services segment, and supports the evaluation of the performance indices of the global or regional grid $[13,18]$. The typical navigation system performance indices are shown in Table 1.

These analyses basically cover all aspects of the performance of the GNSS. The analytical tasks can be further subdivided into several subtasks; and the development, integration, and analysis of the GNSS model has more strict requirements.

1.Reusability of the simulation model. Any task not only depends on the specific mathematical model and data in some fields, but also on the model and data of other fields, thus the reusability of the simulation models is indispensable in reducing the difficulty of model integration in the GNSS simulation.

2.Scalability of the integration framework. To support the specific experiment based on the reuse of modes, algorithms, and data, the integration framework of simulation requires flexibility and configuration. Different models and algorithms can be interchangeable with other models under the analysis type with the framework.

3.Efficiency of model development. The simulation system has to provide the basic model elements and the common support structure, which will support easily the 
Table 1: Typical navigation system performance indices

\begin{tabular}{|c|c|}
\hline Performance indexes & Analyses \\
\hline Visibility & $\begin{array}{l}\text { Global or Regional Visibility } \\
\text { Receiver to Satellite Visibility } \\
\text { Ground Segment to Satellite Extended V isibility }\end{array}$ \\
\hline Coverage & $\begin{array}{l}\text { Depth of Coverage } \\
\text { Ground Segment to Satellite Inverse Depth of Coverage } \\
\text { Independent Integrity Path Coverage }\end{array}$ \\
\hline Geometry & Receiver to Satellite Geometry \\
\hline DOP & $\begin{array}{l}\text { Global or Regional DOP } \\
\text { Global or Regional Availability of DOP } \\
\text { Global or Regional Continuity of DOP }\end{array}$ \\
\hline Navigation Performance & $\begin{array}{l}\text { Global or Regional NSP } \\
\text { Global or Regional Availability of NSP } \\
\text { Global or Regional Continuity of NSP }\end{array}$ \\
\hline Integrity & $\begin{array}{l}\text { Global or Regional Integrity } \\
\text { Global or Regional Availability of Integrity } \\
\text { Global or Regional Continuity of Integrity }\end{array}$ \\
\hline
\end{tabular}

development model of different fields, and reduce the difficulty of developing the model. Thus, it is necessary to standardize the development and integration model.

4.Usability of system configuration. The GNSS simulation should establish the system model's architecture with the characteristics of the satellite navigation system consisting of the space segment, user segment, ground segment, and environment segment, so that simulation users can modify and adjust the test scenario in their preferred way.

According to the characteristics of the aforementioned requirements and the SMP2 specifications, the model framework, execution framework, and integration framework of the GNSS should be established reasonably, and will support numerous performance simulations and analyses. Fig.1 shows the structure of the model components of the GNSS, which includes every key model and core algorithm of different segments. In developing these models and algorithms, different organizations and users may not use the same tools and implementation methods, but the interface of models and algorithms can be consistent with SMP2 specification, so it is possible to reuse and transplant the model and algorithm.

Based on the SMP2 model development specifications, it is necessary to analyze the running process of the GNSS simulation from the perspective of the user. The process can be divided into developing the simulation model, developing the model framework, configuration scenario, data logging and display, and running set and analysis.

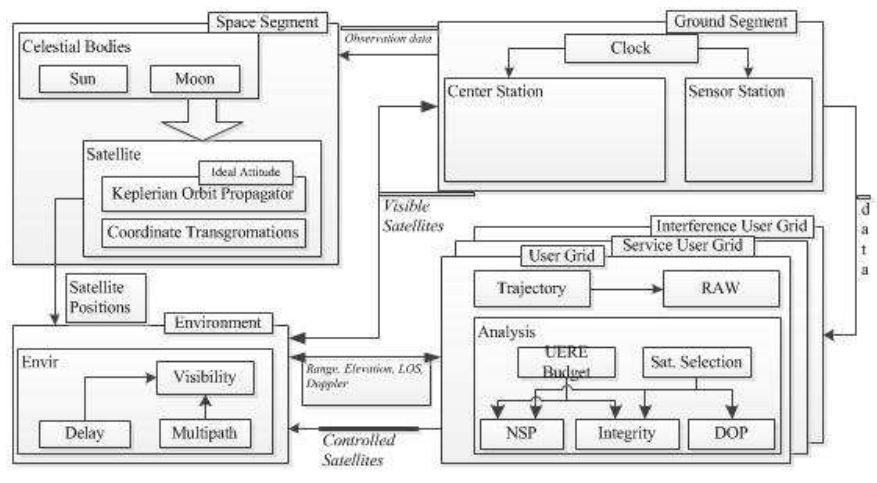

Fig. 1: Structure of the model components of GNSS simulation.

\section{Model Integration Framework of GNSS based on Portability}

According to the aforementioned analysis, the GNSS simulation should develop a model integration framework after the development of domain models. The core idea is to use the SMP2 specification and model reuse method to support the development, testing, and integration of models for the satellite navigation system's engineering level to ensure the consistency of the model development and task analysis.

The space segment of the aforementioned model structure consists of navigation satellite constellation models and celestial models. The ground segment includes the ground station grid model and monitoring of 
the station grid model. The user segment includes a different user grid model. The constellation model consists of a number of satellite models, and the ground station grid model consists of different types of user models.

Based on the navigation performance analysis, the model or algorithm required by different analyses is not the same, which requires the model framework to have good reusability and integration. For example, during the visibility analysis, the environmental segment model has to obtain the positions of satellites and users in the current epoch, then calculate the observed elevation angle to determine the satellite's visibility. In analyzing the Dilution of Precision (DOP) and the Navigation System Precision (NSP), the user grid model has to obtain the visible satellites in the current epoch, then choose the appropriate satellite selection algorithm, and calculate the various DOP and NSP values by using the appropriate budget method of the User Equivalent Range Error (UERE). In the aforementioned simulation, the basic models and algorithms are consistent and can be directly reused, and they also have to be quickly replaced for the validation and evaluation of key algorithms. Thus, it is necessary to establish a unified simulation model integration framework to support the reuse and integration of the various models and algorithms of the navigation system. For the component-based and interface-based SMP2 design method, a simplified portability navigation simulation model integration framework is shown in Fig.2.

In the figure, the SatelliteModel and StationModel represent the domain model of the satellite model and station model, which depicts the design algorithm interface by SMP2 specification, such as Inte_Satellite, Inte_Station, and others. The other models can refer to these models by accessing their interfaces, such as Inte_Satellite, which provides the satellite's health status, the orbital elements, and so on. Some domain model users can use certain models or algorithms to replace some of the models. For example, the environmental model can select a different delay correction algorithm for Ionospheric and Tropospheric simulation, which include the Egnos algorithm, Hopfield algorithm, Saastmanion algorithm, and others. SatellitesContainer and StationsContainer are two types of containers based on the SMP2 specifications, and support the other model entities to query and manage these entities. Inte_Ref_

Satellite and Inte_Ref_UserGrid provide references of the interface for domain models. These references may be multi-reference, for example, EnvironModel is an environmental model, and Inte_Ref_UserGrid is a one-to-many reference from the EnvironModel to the UserGridModel. The RecorderModel and DOPRecorderModel are functional models, and indicate different recording models. In oriented performance simulation analysis, these recording models have to provide the interface to the logging simulation data in the analysis.

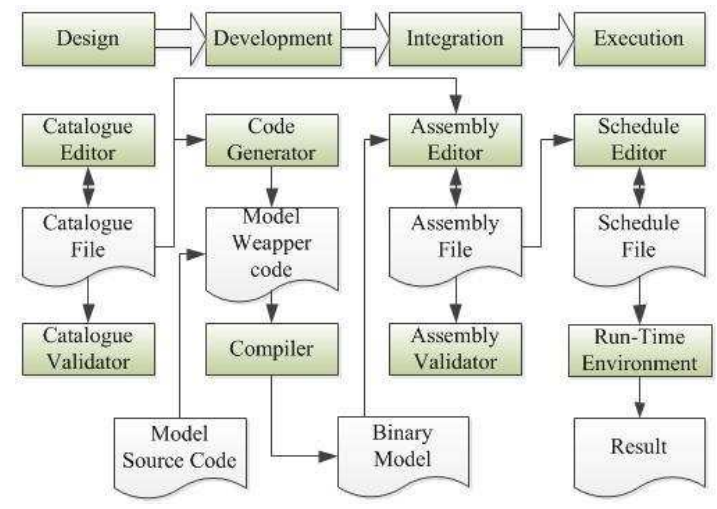

Fig. 3: Application architecture of navigation simulation based on SMP2.

The method of separation of application of the model framework and the simulation scenario framework will help the domain user focus on the familiar navigation domain problems, rather than the specific implementation details of the model framework. The simulation users focus more on the development of the application platform or framework, not the details of the model or algorithm. Based on the application model framework, the corresponding simulation scenario framework can be created with various navigation performance simulation analysis tasks. During the NSP analysis, the satellite model, user grid model, environment model, and NSP recording model, and some interfaces, the references in the framework can be assembled with some scenarios, and then an instance of the scenario can be formed for execution.

\section{Application Architechture and Test of GNSS based on SMP2}

According to the aforementioned integrated model framework, the simulation application development and test architecture based on SMP2 for GNSS can be divided into simulation model design, development, integration, and execution of four stages. Fig.3shows the application architecture based on SMP2.

1.Model design of navigation simulation based on SMP2

The main task of the model design phase of navigation simulation is designing the SMP2 Catalogue file based on the SMDL and model design documents. Simulation users can design models with class-based, interface-based, component-based, event-based, and dataflow-based design approaches of the SMP2, then can obtain the model's information, including the object structure, combination structure, and interaction structure. The oriented SMP2 model integration framework, the Platform Independent Model (PIM), consists of a satellite 


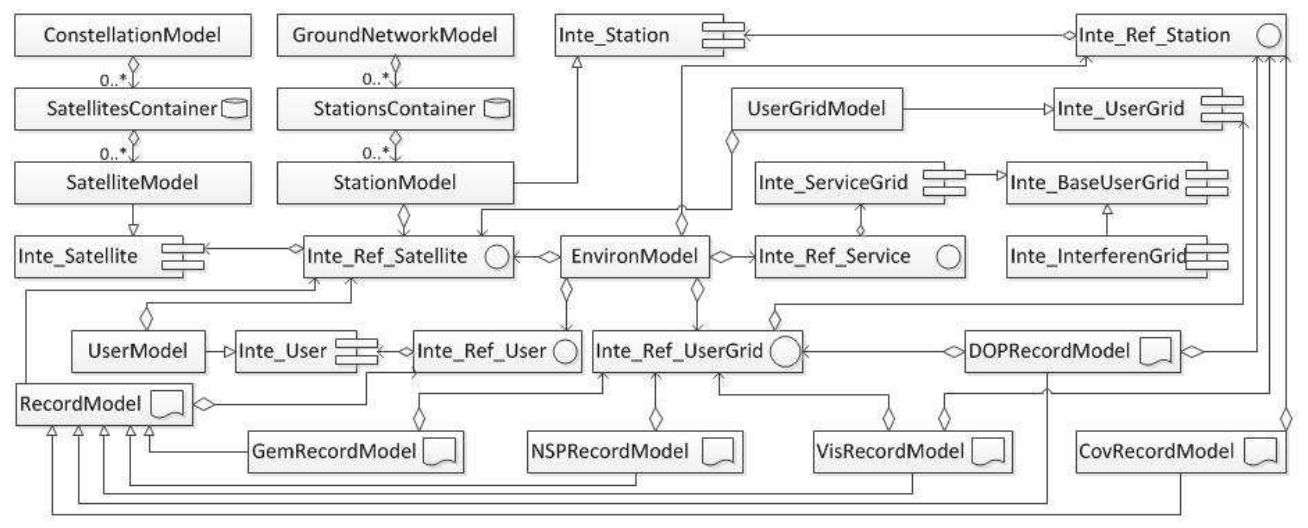

Fig. 2: Model integration framework ofnavigation system oriented-portability.

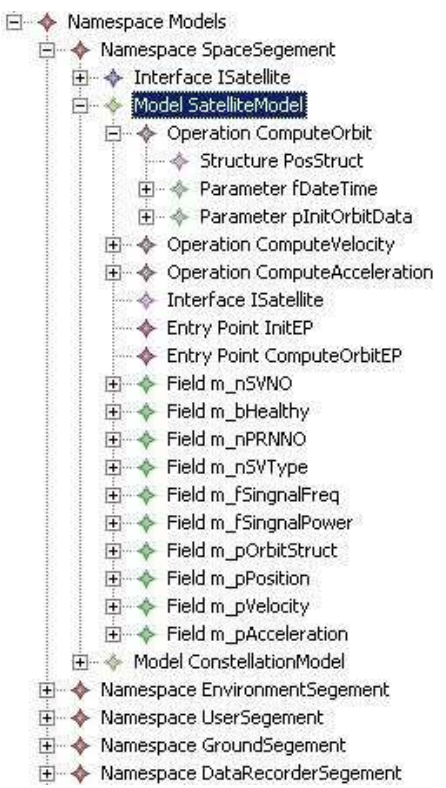

Fig. 4: Satellite Model of PIM.

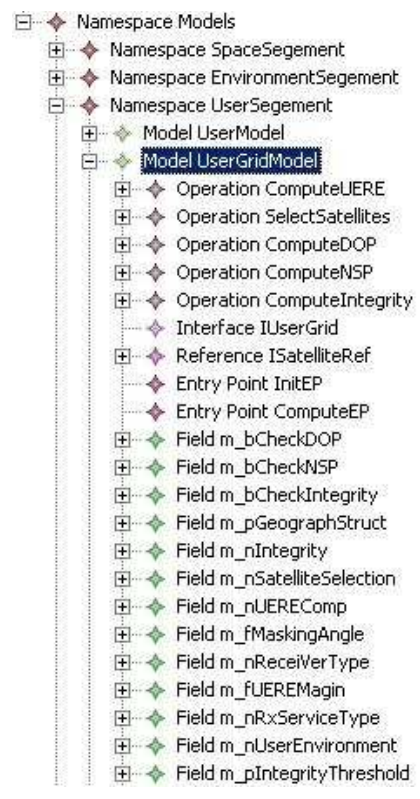

Fig. 5: User Grid Model of PIM. model, constellation model, environment model, user model, user grid model, ground station model, various types of analysis record models, and so on. Using component-based model design approaches, we designed the PIM for the GNSS simulation, and the model's composition, interface, reference relationship, and containment relationship was established in accordance with the aforementioned model integration framework. Fig.4,Fig.5 shows the PIM models for the satellite and user grid. This phase is intended to create the PIM.

\section{Model development of navigation simulation}

\section{based on SMP2}

The main task of the model development phase of navigation simulation is that it translates models from Catalogue file into a model framework code, which transforms the PIM into the PSM, and designs a specific algorithm in the framework. Finally, a compiler is necessary to generate binary models from sources. Based on $\mathrm{C}++$, the JAVA mapping specification provided by SMP2, the PIM would automatically generate the code framework of the navigation simulation model, and add the domain algorithm into the function or reference a dynamic-link library. Finally, the framework code is automatically compiled into binary model libraries, which are PSM components for the execution of the navigation system simulation. The navigation system simulation involves a large number of algorithms such as coordinate system transformation, Julian day calculation, satellite orbit determination, visibility computing, DOP calculation, and so on, which can be compiled in a $\mathrm{C}++$ 
LLink $x s i$ : type="assemb $1 \mathrm{y}:$ InterfaceL ink"

UserGrid"

<Reference $\times$ link: href="SVS. catalogue\#"SVS. Mode1s.RecorderMode1. IUserGridRef" $\times 1$ in

$<$ Provider

$</$ Link $>$

Fig. 6: Relationship of interfaces.

static-link library or dynamic-link library based on the interface relationship defined in the model integration framework. Such algorithms can be replaced quickly as long as they provide consistent interfaces, without the need to recompile the entire model code.

\section{Model integration of navigation simulation based on SMP2}

The main task of the model integration phase of navigation simulation is that it generates the Assembly file, which contains the configuration information and assembly relationships of the model instances by using instance generation, configuration, and integration of the model under the constraints of the Catalogue based on the SMDL. According to the navigation simulation applications demand, simulation users can assemble the models, add the assembly information of models, and then create the PIM-RTC components. Different navigation performance simulation analysis tasks involve different models, and it is necessary to define assembly structure and scenario, so that makes the model reuse and facilitate the integration of different application requirements. During the regional NSP analysis, it is necessary to assemble the constellation model, multi-satellite model, environment model, user grid model, and others, which can be assembled with the interface relationship of these instances as shown in Fig.6.

\section{Model execution of navigation simulation based on SMP2}

The main task of the model execution phase of the GNSS simulation is that it generates the Schedule file, which arranges the scheduling strategy of the navigation model's instance based on the Assembly file and SMDL. The SMP2-based simulation engine will run with the Schedule file, the Assembly file, and the binary model of the navigation simulation as input. The Schedule file defines a number of simulation tasks and events, including the PSM-RTC's information such as the scheduling sequence of the models, the simulation step, and runtime. The simulation task is triggered by a series of task events. The simulator components are used to implement the function of the SMP2 service components, resolve the Catalogue and Assembly files, and then drive the simulation running. During regional DOP analysis, the schedule file has to create two simulation tasks: initialization and computing. The former refers to the initialization entry point of the DOP model instance, and the latter refers to the entry point for orbital determination, updates the Greenwich Mean Time of each

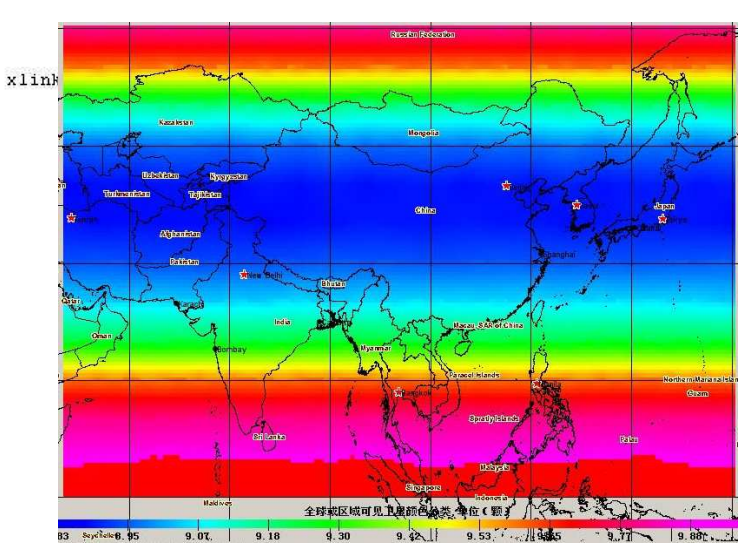

Fig. 7: Regional Visibility Analysis results of navigation system simulation based on SMP2.

satellite instance in turn, then calculates the satellite orbit parameters.

5.Model test of navigation simulation based on SMP2

By using the aforementioned method, we study the performance simulation of the GALILEO system. Based on the navigation performance indices, we define $50^{\circ} \mathrm{E}-150^{\circ} \mathrm{E}, 0^{\circ} \mathrm{N}-60^{\circ} \mathrm{N}$ as an analysis region, and $5^{\circ}$ as grid resolution. The analysis includes visibility, geometry, coverage, DOP, NSP, and others. The satellite orbit determinatiohn algorithm is a Keplerian two-body orbital model, and the simulation cycle is a constellation regression cycle. The simulation step lasts 300 seconds, and the algorithms involved in the model include GPS and GALILEO.

Based on the SMP2 method, we design and develop a software that supports the SMP2-based model development and simulation. The result of the test are reported in figures. Fig.7 is a distribution diagram of the number of visible satellites in this region of GALILEO, which are approximately eight to nine. Fig. 8 is a distribution diagram of the number of ground stations in view at each time step in this region, and most of the distribution is approximately from seven to nine. Fig.9 is a distribution diagram of the mean GDOP values in this region; the values range from $1.91 \mathrm{~m}$ to $2.02 \mathrm{~m}$, and the mean value is $1.93 \mathrm{~m}$. Fig.10 is a distribution diagram of the maximum PNSP values in this region; the values range from $2.41 \mathrm{~m}$ to $2.86 \mathrm{~m}$, and the mean value is 2.65 $\mathrm{m}$. These results are consistent with the data provided by the ESA.

\section{Conclusions and Future Work}

Based on SMP2 specification, we studied the model framework of the GNSS simulation, and proposed an SMP2-based model integration framework for the GNSS, then develop and integrate the models to support the 


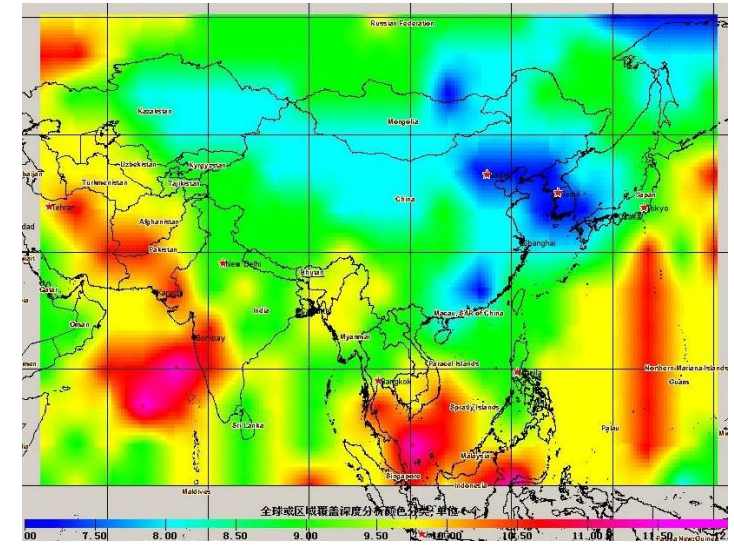

Fig. 8: Regional Coverage Analysis results of navigation system simulation based on SMP2.

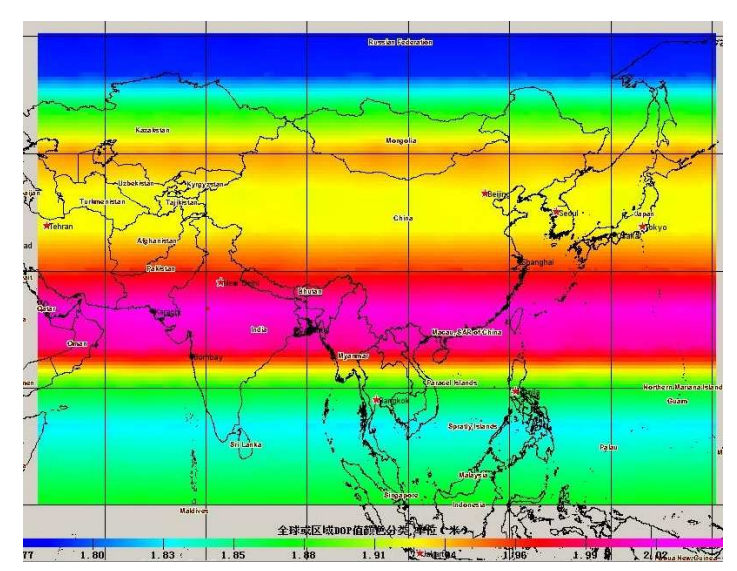

Fig. 9: Regional GDOP Analysis results of navigation system simulation based on SMP2.

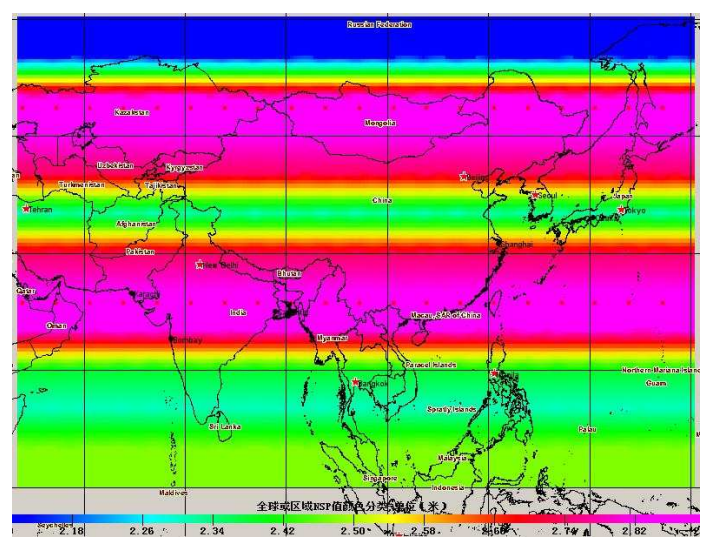

Fig. 10: Regional PNSP Analysis results of navigation system simulation based on SMP2.

performance analysis of the GNSS. Performance analysis by using traditional methods is closely related to the specific requirements of domain or expertise. When the constellation configuration is changed, or the accuracy and approach of the algorithm is changed, we have to re-develop the model, and the algorithms and models tend to lack reusability and portability. Using the proposed method, the domain staff will focus on the development of the domain model, and is not concerned about the simulation platform, so that the developed model can not only support the demonstration program but can also verify the performance impact of the different algorithms or models. The navigation simulation model based SMP2 specification are more reusable and portable.

Our next study will focus on software engineering-oriented SMP2 and the integration of various behavior modeling formalisms.

\section{Acknowledgement}

The authors would like to thank the reviewers of this paper for their valuable comments and advice. This research project was partly supported by the National Science Foundation of China.

The authors are grateful to the anonymous referee for a careful checking of the details and for helpful comments that improved this paper.

\section{References}

[1] Pidgeon A., NASA/ESA Workshop on Product Data Exchange, 7, 210-218 (2004).

[2] Wang Chao, Song Lili, Li Qun, Proceedings of the International Conference on Computational Intelligence and Software Engineering, 5, 235-241 (2010).

[3] Wang Chao, Li Qun, Wang Weiping, 2011 International Conference on Electric Information and Control Engineering, 3, 125-129 (2011).

[4] Peter Ellsiepen, Peter Fritzen, Simulation model portability Technical Report, European Space Oerations Centre, (2005).

[5] Paul A. Fishwick, Simulation Model Design and Execution, Prentice-Hall Inc, New Jersey, (1995).

[6] Peter Fritzen,The Royal Aeronautical Society "RaeS Simulation of On-board Systems" Proceedings, 6, 136-142 (2004).

[7] B. Zhang, Z. Juan, Modeling User Equilibrium and the Day-to-day Traffic Evolution based on Cumulative Prospect Theory, Information Sciences Letters, 2, 9-12 (2013).

[8] Hou Hongtao, Zhou Hongwei, Li qun, Journal of System Simulation, 22, 1350-1354 (2010).

[9] Wang Li,A Study On Issues Of Overall Design For Satellite Navigation System, PhD thesis, Natinoal University of Defense Technology, (2007).

[10] Su Nianle, Yu Wenguang, Hou Hongtao, Proceedings of 7th IEEE East-West Design and Test Symposium, 7, 656-661 (2009).

[11] ChenShu, WuGuoQing, YeJunMin, A Domain Oriented Approach for Network Software Requirement Modeling, Information Sciences Letters, 1, 21-39 (2012). 
[12] F. Salvadori, C. S. Gehrke, M. de Campos, P. S. Sausen, A. C. Oliveira, A Hybrid Network Architecture Applied to Smart Grid, International Journal of Computing and Network Technology, 1, 45-59 (2013).

[13] John P. Stenbit, Global positioning system standard positioning service performance standard, (2008).

[14] Zhang Wei, Yu Yongli, Zhao Xinhui,Proceedings of 2011 9th International Conference on Reliability, Maintainability and Safety, 9, 774-778 (2011).

[15] Lei Yonglin, Su Nianle, Li Jingjie,System Engineering Theory and Practice, 30, 899-908 (2010).

[16] Moon Soo Young and Seo Hee Suk, Appl. Math. Inf. Sci., 6, 286-289 (2012).

[17] Lei Yonglin, Wang Weiping, Li Qun, Simulation Modelling Practice and Theory, 17, 1690-1709 (2009).

[18] Zainuddin Z. and Shuaib M. M. A., Appl. Math. Inf. Sci. 5, 53-64 (2011).

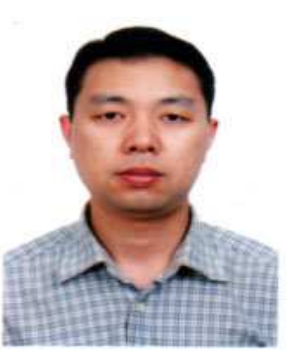

Hou Hongtao is currently working towards the Ph.D. degree in School of Information System and Management in National University of Defense Technology.His current research interests include flexible simula-tion theory, virtual simulation, distributed and parallel simulation, satellite navigation system simulation and system of systems engineering.

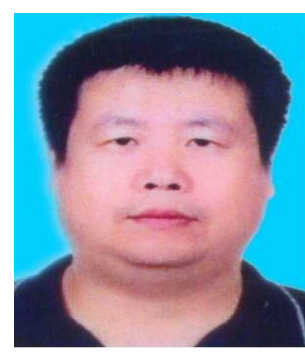

Li Qun is Professor of National University of Defense Technology. He received his Ph.D. degree in control science and engineering from National University of Defense Technology in 1999. His current research interests include flexible simulation theory, simulation for operational effectiveness evaluation and system of systems engineering.

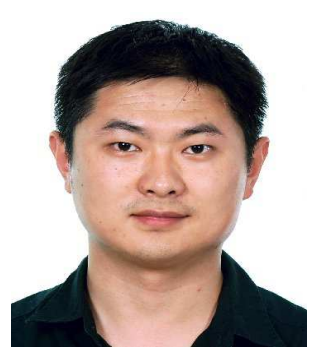

Wang Chao is currently working towards the Ph.D. degree in School of Information System and Management in National University of Defense Technology.His current research interests include flexible simula-tion theory, distributed and parallel simulation and satellite navigation system simulation. 